\title{
Resultados de la implantación de un método de innovación docente en el aula. Flip-teaching.
}

\section{Raquel Giménez Ibáñez y Emma Barelles Vicente ${ }^{\mathrm{b}}$}

${ }^{a}$ Universidad Politécnica de Valencia, raquel@raquelgimenez.es y ${ }^{b}$ Universidad Politécnica de Valencia, ebarelle@arq.upv.es.

\begin{abstract}
The method of educational innovation that is implemented is based on the Flip-Teaching or inverse class and this one develops with the inversion of the roles protagonists in the classrooms. The teacher prepares content for the students, the students review them before class and during the class working on these concepts totally practical way. In this way the student practices from the moment and doubts arise in the classroom where the teacher is and can help you solve them. The method is implemented by the need that is observed in the subject to change the system because it is about content that must be learned by means of the practice. This article shows the results obtained after having implemented this system and analyzing the differences appeared over previous years.
\end{abstract}

Keywords: teaching, educational innovation, flip-teaching

\begin{abstract}
Resumen
El método de innovación educativa que se implanta está basado en el FlipTeaching o clase inversa y se desarrolla con la inversión the los roles de los protagonistas en las aulas. El profesor prepara contenidos a los alumnos, los alumnos los revisan antes de la clase y durante la clase se trabaja sobre esos conceptos de manera totalmente práctica. De esta forma el alumno practica desde el primer momento y las dudas surgen en el aula donde el profesor se encuentra y puede ayudarle a resolverlas. El método se implanta por la necesidad que se observa en la asignatura de cambiar el sistema por tratarse de unos contenidos que como mejor se aprenden es practicándolos. Este artículo pretende mostrar los resultados obtenidos después de haber implantado este sistema y las diferencias aparecidas con respecto a años anteriores.
\end{abstract}

Palabras clave: docencia, innovación educativa, flip-teaching 


\section{Introducción}

El método de innovación educativa que se implanta está basado en la metodología Flip Teaching. Se pretende involucrar al alumno en su propio aprendizaje, de manera que se convierta en el protagonista principal y participe de forma completamente activa en la adquisición de nuevos conocimientos a través del trabajo activo desarrollado en el aula y organizado por el profesor.

La asignatura trata sobre el estudio de edificios con algún tipo de lesión. Los objetivos fijados en la asignatura es que los alumnos consigan las herramientas necesarias para poder diagnosticar las lesiones que sufre el edificio, las causas que las pueden producir y cómo se debe intervenir adecuadamente para poder eliminarlas y que no vuelvan a aparecer. Para ello deben basarse en los conocimientos adquiridos en asignaturas cursadas con anterioridad.

\section{Objetivos}

Habiendo detectado que la asignatura tiene un problema, ya que la mayoría de alumnos no alcanzan los objetivos fijados, se implanta este sistema con el fin de conseguirlos y se fijan nuevos hitos que permitirán alcanzarlos.

El alumno debe aprender a ser autonómo a la hora de afrontar los problemas. El profesor siempre estará presente para poder apoyarle en las dudas que puedan surgir, pero es el alumno el que debe comenzar a trabajar de manera independiente. El profesor le facilita para ello una serie de contenidos con anterioridad al inicio de las clases, para que tenga conocimientos y herramientas de trabajo para ello. Todos los actos en los que participa el alumno son evaluados para fomentar el feedback entre el profesor y el alumno. Por ello, Este nuevo sistema no permite el absentismo porque de no acudir a las clases, los alumnos no reciben la nota correspondiente, lo que hace que aumente la asistencia.

El profesor puede hacer un seguimiento continuo de los avances del alumno a través del contacto directo que tiene con este en las aulas. De esta manera se puede comprobar que los conocimientos alcanzados son correctos. Esto se consigue al tutorizar de manera directa con el alumno, guiándole en la resolución de los problemas al sustituir la clase magistral por el trabajo directo en el aula. Las experiencias anteriores demuestran que la docencia tradicional de clase magistral y trabajo en casa supone un impedimento para poder llegar a alcanzar los objetivos fijados.

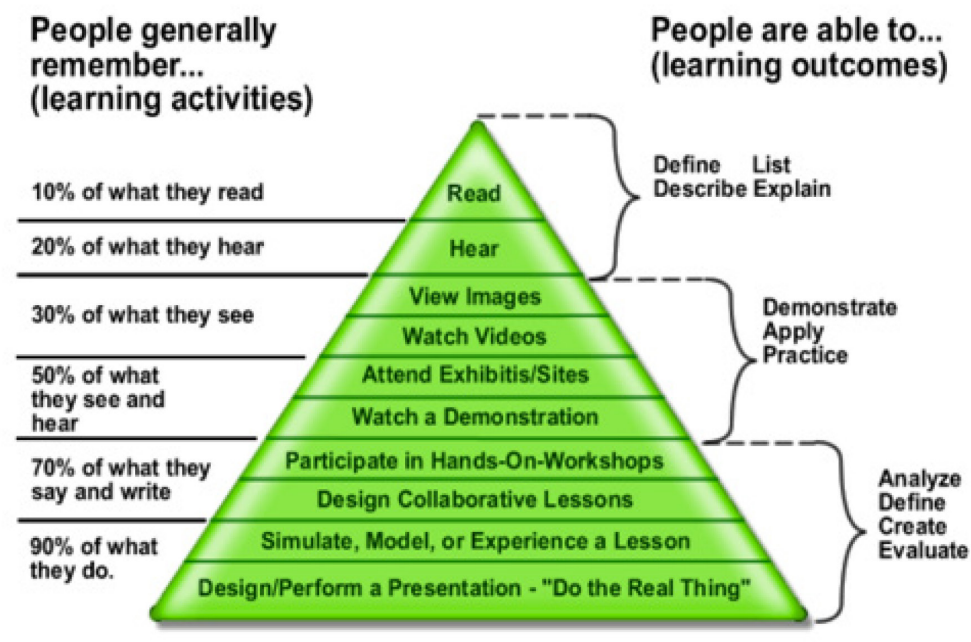

Fig. 1 Esta figura se basa en el cono de la experiencia de Edgar Dale, observamos cómo varia la cantidad de información que recuerda un alumno, en función de la actividad de aprendizaje llevada a cabo 


\section{Desarrollo de la innovación}

La asignatura se divide en clases de teoría y práctica. Las clases de teoría tienen una duración de 2 horas y las clases de práctica de 1,5 horas. Al inicio de la semana el profesor cuelga en la plataforma online de la Universidad PoliformaT, los contenidos que ha preparado para ayudar al alumno a enfrentarse a las prácticas que se realizarán en la siguiente clase. Estos contenidos son, videos realizados por él o no, relacionados con el tema a trabajar durante la semana, artículos técnicos relacionados, apuntes y todo tipo de documentación que considera necesaria para poder afrontar la siguiente clase. El alumno debe visionar el video, contestar a las preguntas relacionadas con el tema que se encuentran al final y preparar la documentación necesaria para acudir a la siguiente clase y resolver la práctica planteada.

En la siguiente clase, que será de teoría, el profesor dedica los primeros minutos a resolver las dudas que hayan podido surgir durante el visionado de la documentación colgada. A continuación enuncia la práctica a desarrollar y los alumnos comienzan a trabajar en grupos de tres personas. De esta manera también se fomenta el trabajo cooperativo.

Hacia el final de la clase los alumnos exponen sus resultados de manera pública y así se discute sobre los resultados obtenidos y se explica la solución correcta. Todos tienen una nota que dependerá del desarrollo de trabajo realizado en el aula como respuesta a su esfuerzo. Pero que no es resultado de la valoración de su adquisición de conocimientos, ya que el excesivo número de alumnos nos impide su evaluación individualizada.

En la siguiente clase, que se dedica a la práctica, los alumnos trabajan sobre el Proyecto de curso, también de manera cooperativa en grupos de seis personas y el profesor va resolviendo cualquier duda que surge. En este caso también se puntúa el trabajo realizado en clase.

El Proyecto de curso, se entrega al final del cuatrimestre, pero con este método de trabajo se consigue que los alumnos se organicen y trabajen sobre él de manera semanal, no teniendo acumulación de trabajo unos días antes de la entrega final. Que es lo que ocurría con el sistema tradicional utilizado con anterioridad.

\section{Resultados}

Una vez concluida la primera experiencia realizada con esta nueva metodología se puede hacer balance de los resultados obtenidos. Los vamos a analizar desde el punto de vista de notas alcanzadas, de número de aprobados, de asistencia (que era un problema anteriormente) y también la percepción por parte del alumno después de analizar los resultados de las encuestas.

A continuación se muestran dos imágenes con las estadísticas tanto de las notas obtenidas por los alumnos, como de los alumnos aprobados, en las que se puede ver la diferencia sustancial que existe con el año anterior. 


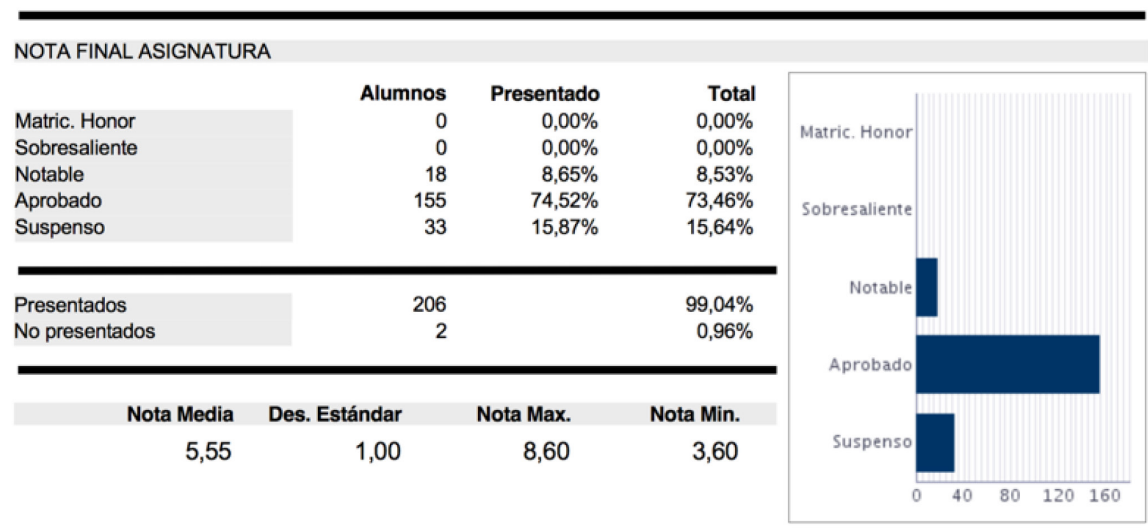

Fig. 2 Estadísticas finales del curso 2014-2015

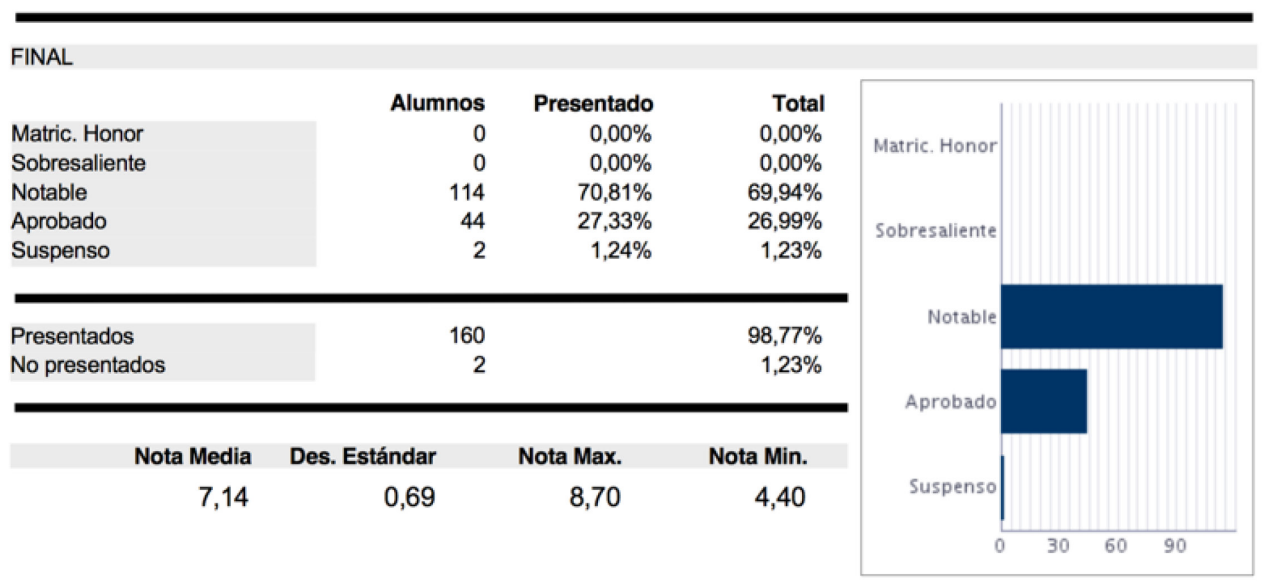

Fig. 3 Estadísticas finales del curso 2015-2016

En estas figuras se puede observar una diferencia muy grande en cuanto a la nota final alcanzada por la mayor cantidad de alumnos. Mientras que en este curso, impartido con la nueva docencia la mayor cantidad de alumnos tienen un notable, en el año anterior impartido con la docencia tradicional la mayor cantidad de alumnos tienen un aprobado.

El número de alumnos suspendidos es también muy llamativo, 33 del curso 2014-2015 frente a los 2 de este curso.

La asistencia a las clases ha sido del $98 \%$ durante todo el curso, sin excepción. Mientras que el curso anterior, se comenzaba con una asistencia relativamente alta, del $90 \%$, que iba decayendo conforme avanzaba el curso y se les acumulaban los exámenes y entregas de trabajos de otras asignaturas, hasta situarse en un $40 \%$ en el ultimo mes.

En cuanto a la percepción del alumno, en líneas generales, se encuentra satisfecho con el nuevo sistema, según los datos extraídos de la encuesta realizada por parte de la Universidad. Sobre todo los alumnos que cursaron la asignatura el año anterior y comparan

(c)) EY-NC-ND 2016, Universitat Politècnica de València 
un sistema con otro son los que más satisfechos se muestran y comentan la importancia del trabajo de clase con el apoyo continuo del profesor. Lo que les facilita la realización de muchas más prácticas que en años anteriores.

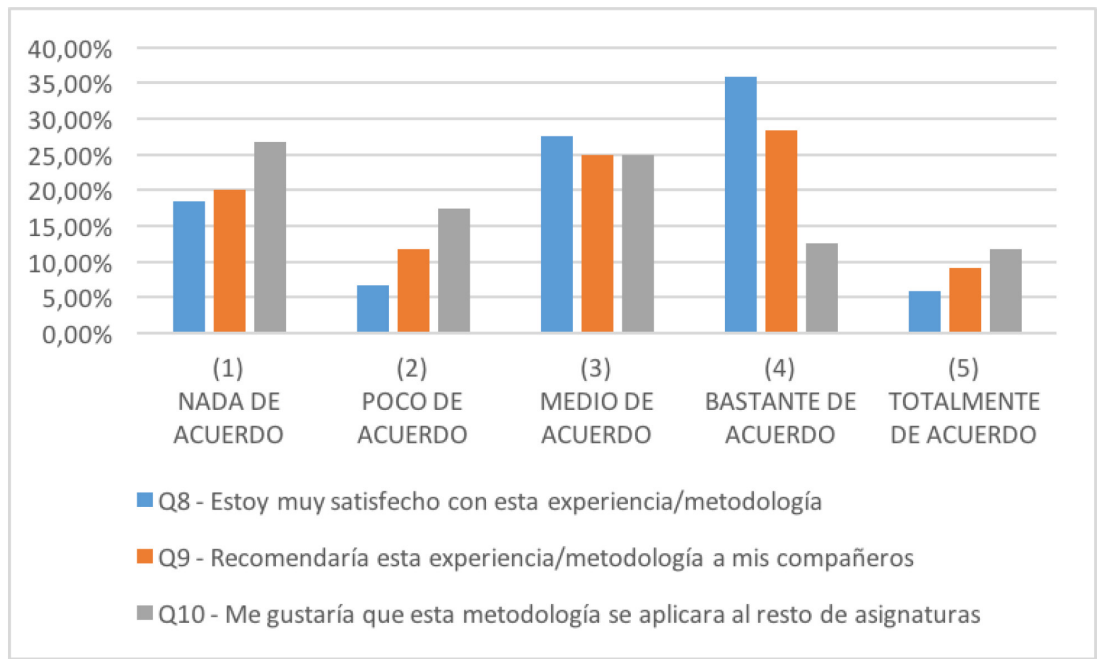

Fig. 4 Resultados de la encuesta realizada al alumno. Grado de satisfacción con el sistema Flip-teaching

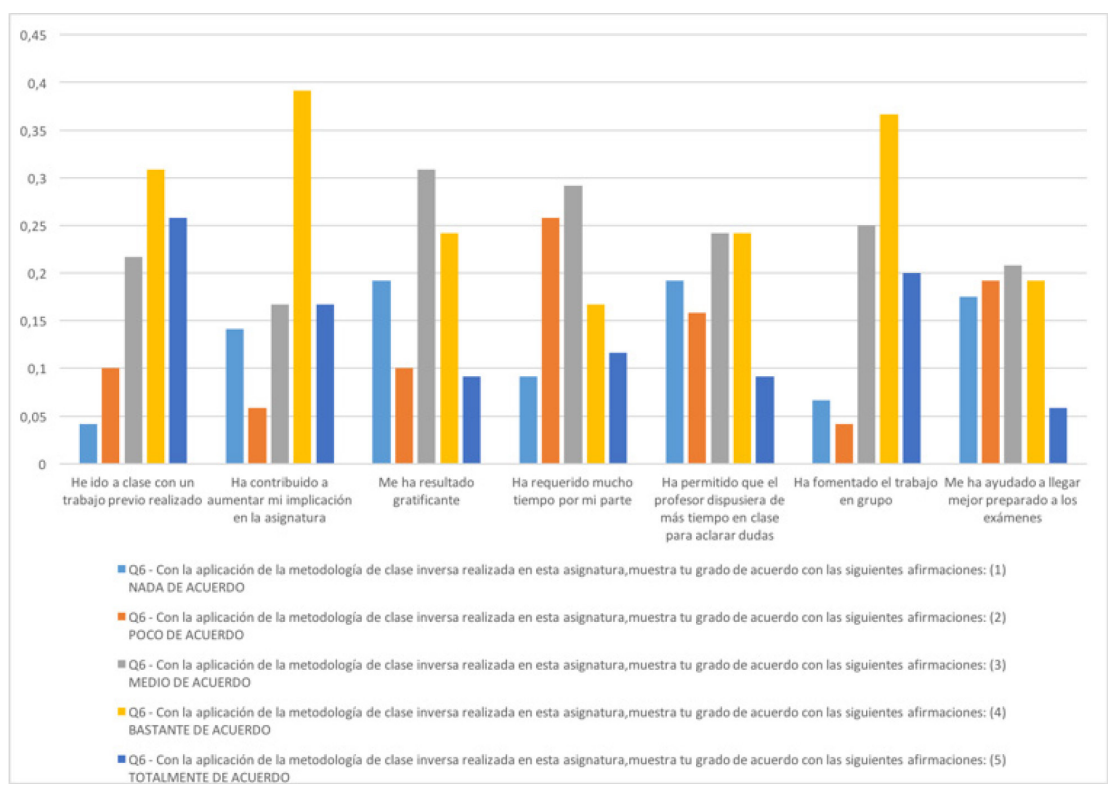

Fig. 5 Resultados de la encuesta realizada al alumno. Grado de satisfacción con el sistema Flip-teaching

\section{Conclusiones}

En todo caso, nuestra percepción que es muy positiva respecto al nuevo sistema, también tiene algunas deficiencias que deben ser mejoradas para el siguiente curso. A continuación se enumeran. Unas tienen que ver con nuestra vivencia directa y otras tienen que ver con los comentarios realizados por los alumnos de manera anónima en la encuesta realizada sobre el tema.

(cc) EY-NC-ND 2016, Universitat Politècnica de València

Congreso In-Red (2016) 
En primer lugar, nuestra percepción nos hace pensar que aun teniendo notas muy altas, la mayoría de alumnos, esto no significa que todos hayan alcanzado los objetivos iniciales de aprendizaje. El hecho de haber puntuado el trabajo de clase cada día ha hecho que todos tengan una nota por el simple hecho de haber asistido. Esto ha motivado al alumno a asistir a clase de manera regular, pero ha premiado su asistencia con una nota. Y no se ha reflejado en la calidad de los trabajos finales. Por esta razón, se van a introducir dos modificaciones, una de las cuales será la de realizar una prueba tipo test al final de cada clase de teoría en la que se pregunte sobre los conceptos tratados en el video creado por el profesor y en los conceptos trabajados durante esa clase, y además se planteará una prueba objetiva al final del cuatrimestre que mida el grado en el que se ha alcanzado la adquisición de conocimientos.

También se ha decidido incluir una primera parte en la clase de teoría que desarrolle conceptos clave, sin necesidad de ser el alumno el que plantee las dudas, dada la pasividad de este observada durante el curso.

En cuanto a la percepción de los alumnos, ellos demandan mayor amplitud de conocimientos en los materiales sobre todo visuales que se les facilitan. Por lo cual pensamos mejorar los videos de manera sustancial, incluyendo algunos que desarrollen casos de manera práctica.

Una cosa importante a tener en cuenta es el número de alumnos que conforman cada grupo. El hecho de ser grupos muy numerosos, en algunos casos 40 personas dificulta la atención que se les puede dar a cada uno de ellos, lo cual ha suscitado observaciones por parte del alumno. En este sentido, se ha pedido a la jefatura de estudios que limite los grupos a 30 personas para que esta atención pueda ser más equitativa.

\section{Referencias}

BAKER, J.W. «The classroom flip » : Using web course management tools to become the guide by the side. «Selected Papers from the 11 th International Conference on College Teachig and Learning. 2000

BISHOP, JACOB LOWELL, AND MATTHEW A VERLEGER. «The Flipped Classroom: A Survey of the Research » 2013

CUBILLOS, JORGE. «A Comparative Stuy of hybrid versus Traditional Instruction in Foreign Languages » NECTFL Review 60 (2007) 30-38

EDUCAUSE Learning Initiative. « 7 Things you should know about Flippe Classrooms » (2012). GUIDRY, KEVIN R, JORGE CUBILLOS, AND KATHLEEN L. PUSECKER. « The connection between selfregulated learning and student success in a hybrid course » Long Beach, California : n.p, 2013

LAGE, MAUREEN J, GLENN J PLATT, AND MICHAEL TREGLIA. « Inverting the classroom : a gateway to creating an inclusive learning environment. « The journal of economic education 31.1 (2000) : 30-43

MULDROW, KAREN «A new approach to language instruction-flipping the classroom. « The language educator (2013) : 28-31

STRAYER, JEREMY F. «How learning in an inverted classroom influences cooperation, innovation and task orientation » Learning environments research 15.2 (2012) : 171-193. CrossRef. Web. 2 noviembre 2014

TALBERT, ROBERT «Inverted Classroom » Colleagues 9.1 (2012) 1-3

(c)) EY-NC-ND 2016, Universitat Politècnica de València

Congreso IN-RED (2016) 